\title{
The Exponentiated Burr XII Poisson Distribution with Application to Lifetime Data
}

\author{
Ronaldo V. da Silva ${ }^{1}$, Frank Gomes-Silva ${ }^{2}$, Manoel Wallace A. Ramos ${ }^{3}$ \& Gauss M. Cordeiro ${ }^{4}$ \\ ${ }^{1}$ Recife Military School, Recife, PE, Brazil \\ 2 Department of Statistics and Informatics, Federal Rural University of Pernambuco, Recife, PE, Brazil \\ ${ }^{3}$ Federal Institute of Paraíba, João Pessoa, PB, Brazil \\ ${ }^{4}$ Department of Statistics, Federal University of Pernambuco, Recife, PE, Brazil \\ Correspondence: Frank S. Gomes da Silva, Department of Statistics and Informatics, Federal Rural University of \\ Pernambuco, Rua Dom Manoel de Medeiros, s/n, Campus Dois Irmãos, 52171-900, Recife, Pernambuco, Brazil. \\ Tel: 81-3320-6491. E-mail: franksinatrags@gmail.com
}

Received: August 13, 2015 Accepted: August 27, 2015 Online Published: October 9, 2015

doi:10.5539/ijsp.v4n4p112 URL: http://dx.doi.org/10.5539/ijsp.v4n4p112

\begin{abstract}
A five-parameter distribution, called the exponentiated Burr XII Poisson distribution, is defined and studied. The model has as special sub-models some important lifetime distributions discussed in the literature, such as the logistic, log-logistic, Weibull, Burr XII and exponentiated Burr XII distributions, among several others. We derive the ordinary and incomplete moments, generating and quantile functions, Bonferroni and Lorenz curves, mean deviations, reliability and two types of entropy. The order statistics and their moments are investigated. The method of maximum likelihood is proposed for estimating the model parameters. We obtain the observed information matrix. An application to a real data set demonstrates that the new distribution can provide a better fit than other classical lifetime models. We hope that this generalization may attract wider applications in reliability, biology and survival analysis.
\end{abstract}

Keywords: Beta distribution, Burr XII distribution, Maximum likelihood, Observed information matrix, Weibull distribution

\section{Introduction}

The statistics literature has numerous distributions for modeling lifetime data. But many if not most of these distributions lack motivation from a lifetime context. For example, there is not apparent physical motivation for the gamma distribution. It only has a more general mathematical form than the exponential distribution with one additional parameter, so it has nicer properties and provides better fits. The same arguments apply to the BXII distribution, among others.

Zimmer et al. (1998) introduced the three parameter Burr XII (BXII) distribution with cumulative distribution function (cdf) and probability density function (pdf) (for $x>0$ ) given by

$$
G(x ; s, k, c)=1-\left[1+\left(\frac{x}{s}\right)^{c}\right]^{-k}
$$

and

$$
g(x ; s, k, c)=c k s^{-c} x^{c-1}\left[1+\left(\frac{x}{s}\right)^{c}\right]^{-k-1}
$$

respectively, where $k>0$ and $c>0$ are shape parameters and $s>0$ is a scale parameter. If $c>1$, the density function is unimodal with mode at $x=s[(c-1) /(c k+1)]^{1 / c}$ and is L-shaped if $c=1$. If $q<c k$, the $q$ th moment about zero is $\mu_{q}^{\prime}=s^{q} k B\left(k-q c^{-1}, 1+q c^{-1}\right)$, where $B(p, q)=\Gamma(p) \Gamma(q) / \Gamma(p+q)$ and $\Gamma(p)=\int_{0}^{\infty} x^{p-1} \mathrm{e}^{-\mathrm{x}} \mathrm{dx}$ is the gamma function.

The BXII distribution, having as sub-models the logistic and Weibull distributions, is a very popular distribution for modeling lifetime data and phenomenon with monotone failure rates. When modeling monotone hazard rates, the Weibull model may be an initial choice because of its negatively and positively skewed density shapes.

Nevertheless, it does not furnish a reasonable parametric fit for non-monotone failure rates such as the bathtub shaped and unimodal failure rates that are common in reliability and biological studies. 
Several other authors including El-Bassiouny and Abdo (2010), Jayakumar and Mathew (2008), Brito et al. (2014) and Ramos et al. (2015) proposed and developed the structural properties of various generalized Burr XII distributions.

The cdf and the reliability function of the three-parameter BXII distribution can be expressed in closed-form, thus simplifying the computation of the percentiles and the likelihood function for censored data. This distribution has algebraic tails that are effective for modeling failures occurring with lesser frequency than with those models based on exponential tails. Hence, it represents an adequate distribution for modeling failure time data (Zimmer et al., 1998). Shao (2004) discussed maximum likelihood estimation of its parameters and Shao et al. (2004) studied models for extremes based on the BXII distribution with application to flood frequency analysis. According to Soliman (2005), this model generalizes a large number of distributions. Its versatility and flexibility turns it quite attractive as a tentative model for lifetime data.

For an arbitrary baseline cdf $G(x)$, a random variable is said to have the exponentiated-G ("Exp-G" for short) distribution with parameter $a>0$, say $X \sim \operatorname{Exp}-G(a)$, if its pdf and cdf are $H_{a}(x)=G^{a}(x)$ and $h_{a}(x)=a G^{a-1}(x) g(x)$, respectively. Thus, the cdf and pdf of the exponentiated Burr XII (Exp-BXII) distribution is given by

$$
G_{\alpha}(x ; s, k, c)=\left\{1-\left[1+\left(\frac{x}{s}\right)^{c}\right]^{-k}\right\}^{\alpha}
$$

and

$$
g_{\alpha}(x ; s, k, c)=k c s^{-c} \alpha x^{c-1}\left[1+\left(\frac{x}{s}\right)^{c}\right]^{-k-1}\left\{1-\left[1+\left(\frac{x}{s}\right)^{c}\right]^{-k}\right\}^{\alpha-1}
$$

respectively.

We provide four motivations for the proposed lifetime model called the exponentiated BXII Poisson (Exp-BXIIP) distribution. The first is based on failures of a system. Suppose that a system has $N$ serial sub-systems functioning independently at a give time, where $N$ is a truncated Poisson random variable with probability mass function (pmf)

$$
\operatorname{Pr}(N=n)=\frac{1}{\left(\mathrm{e}^{\lambda}-1\right)} \frac{\lambda^{n}}{n !}
$$

for $n=1,2, \ldots$ Let $X$ denote the time of failure of the first out of the $N$ functioning systems defined by the independent random variables $Y_{1} \sim \operatorname{Exp}-\mathrm{BXII}(\alpha), \ldots, Y_{N} \sim \operatorname{Exp}-\mathrm{BXII}(\alpha)$ given by the cdf (3). Then, $X=$ $\min \left(Y_{1}, \ldots, Y_{N}\right)$. So, the conditional cdf of $X$ (for $x>0$ ) given $N$ is

$$
\begin{aligned}
F(x \mid N) & =1-\operatorname{Pr}(X>x \mid N)=1-\operatorname{Pr}\left(Y_{1}>x, \ldots, Y_{N}>x\right) \\
& =1-\operatorname{Pr}^{N}\left(Y_{1}>x\right)=1-\left[1-\operatorname{Pr}\left(Y_{1} \leq x\right)\right]^{N} \\
& =1-\left\{1-\left[1-\left[1+\left(\frac{x}{s}\right)^{c}\right]^{-k}\right]^{\alpha}\right\}^{N},
\end{aligned}
$$

where $s, k, c, \alpha, \lambda>0$. Hence, the unconditional cdf of $X$ is

$$
\begin{aligned}
F(x) & =\frac{1}{\left(\mathrm{e}^{\lambda}-1\right)} \sum_{n=1}^{\infty}\left\{1-\left[1-\left(1-\left[1+\left(\frac{x}{s}\right)^{c}\right]^{-k}\right)^{\alpha}\right]^{n}\right\} \frac{\lambda^{n}}{n !} \\
& =\frac{1}{\left(1-\mathrm{e}^{-\lambda}\right)}\left\{1-\exp \left\{-\lambda\left[1-\left(1+\left(\frac{x}{s}\right)^{c}\right)^{-k}\right]^{\alpha}\right\}\right\} .
\end{aligned}
$$

Then,

$$
F(x)=\frac{1}{\left(1-\mathrm{e}^{-\lambda}\right)}\left\{1-\exp \left[-\lambda G(x)^{\alpha}\right]\right\}
$$

where $G(x)=G(x ; s, k, c)$ is given by (1). We refer to the distribution (6) as the Exp-BXIIP distribution. Proving a new lifetime distribution is always precious for statisticians. The fact that the new model generalizes existing commonly used distributions is also a positive point. 
The survival function associated with $X$ becomes

$$
S(x)=1-F(x)=\frac{1}{\left(1-\mathrm{e}^{-\lambda}\right)}\left\{\exp \left\{-\lambda\left[1-\left(1+\left(\frac{x}{s}\right)^{c}\right)^{-k}\right]^{\alpha}\right\}-\exp (-\lambda)\right\} .
$$

The probability density function (pdf) corresponding to (6) is given by

$$
\begin{aligned}
f(x ; s, k, c, \alpha, \lambda) & =\frac{c k s^{-c} \alpha \lambda}{1-\mathrm{e}^{-\lambda}} x^{c-1}\left[1+\left(\frac{x}{s}\right)^{c}\right]^{-k-1}\left\{1-\left[1+\left(\frac{x}{s}\right)^{c}\right]^{-k}\right\}^{\alpha-1} \\
& \times \exp \left\{-\lambda\left[1-\left(1+\left(\frac{x}{s}\right)^{c}\right)^{-k}\right]^{\alpha}\right\} .
\end{aligned}
$$

Hereafter, a random variable $\mathrm{X}$ with density function (9) is denoted by $X \sim \operatorname{Exp}-\mathrm{BXIIP}(s, k, c, \alpha, \lambda)$. Plots of the density function of $X$ for selected parameter values are displayed in Figure 1.
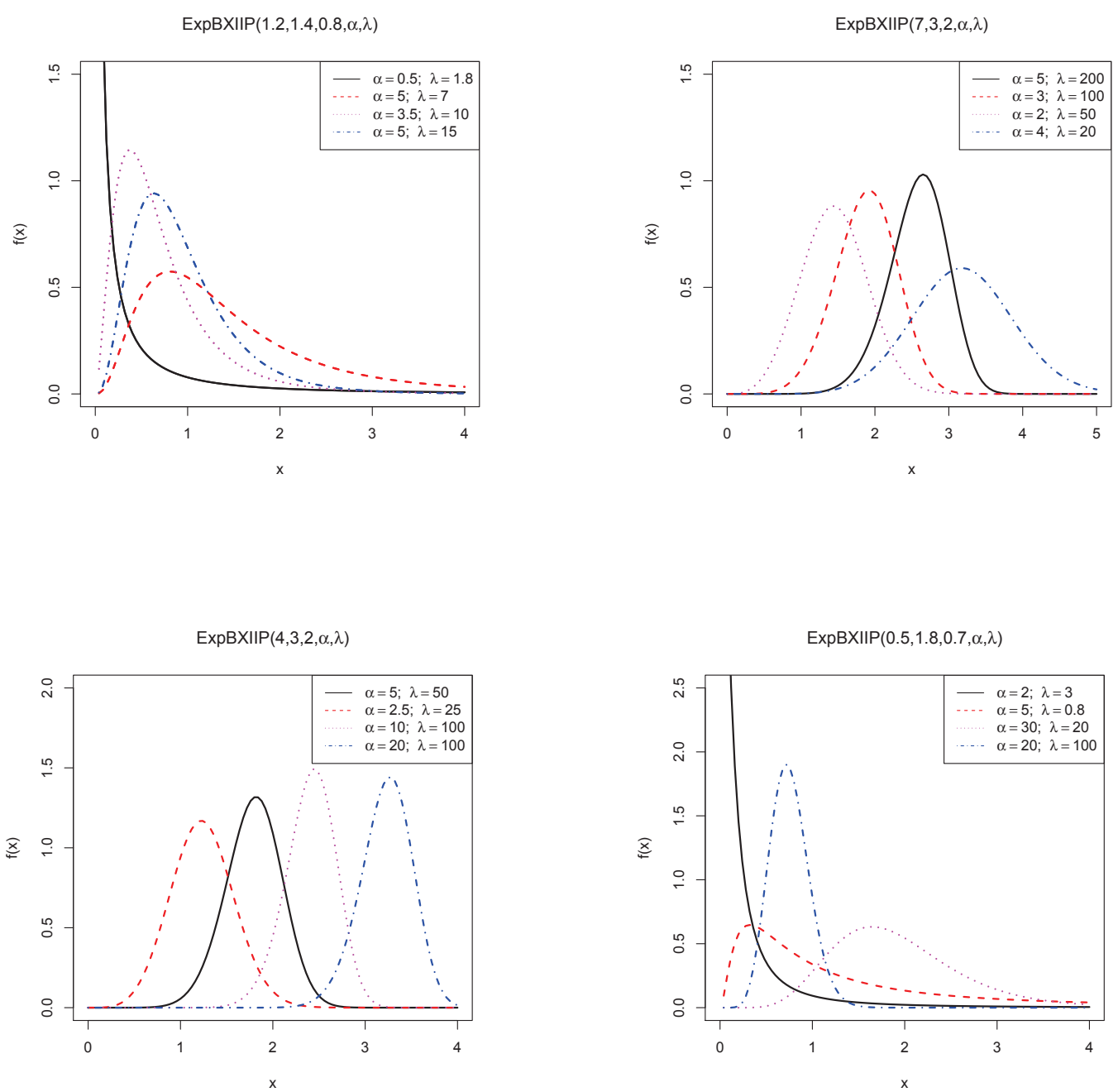

Figure 1. Plots for the Exp-BXIIP density for some parameter values. 
The Exp-BXIIP hazard rate function (hrf) is given by

$$
\begin{aligned}
\tau(x ; s, k, c, \alpha, \lambda) & =\frac{c k s^{-c} \alpha \lambda x^{c-1}\left[1+\left(\frac{x}{s}\right)^{c}\right]^{-k-1}\left\{1-\left[1+\left(\frac{x}{s}\right)^{c}\right]^{-k}\right\}^{\alpha-1}}{\exp \left\{-\lambda\left[1-\left(1+\left(\frac{x}{s}\right)^{c}\right)^{-k}\right]^{\alpha}\right\}-\exp (-\lambda)} \\
& \times \exp \left\{-\lambda\left[1-\left(1+\left(\frac{x}{s}\right)^{c}\right)^{-k}\right]^{\alpha}\right\} .
\end{aligned}
$$

Plots of the hazard rate functions for selected parameter values are displayed in Figure 2.

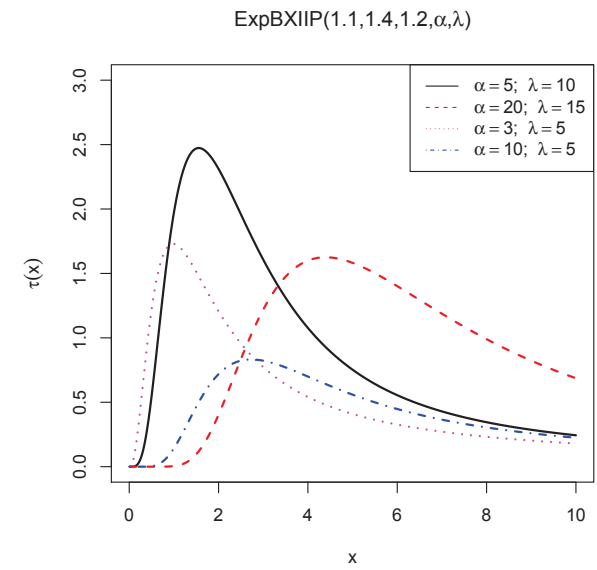

$\operatorname{ExpBXIIP(s,k,c,0.5,2)}$

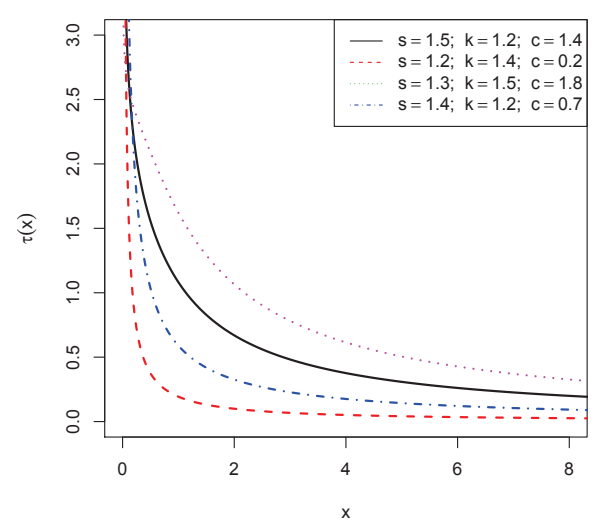

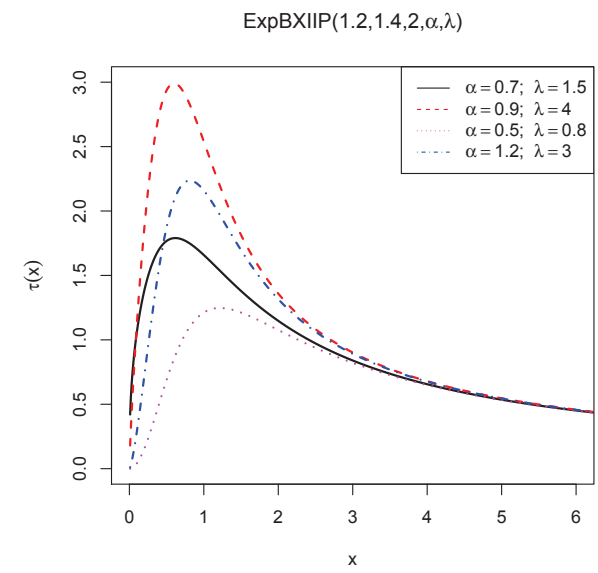

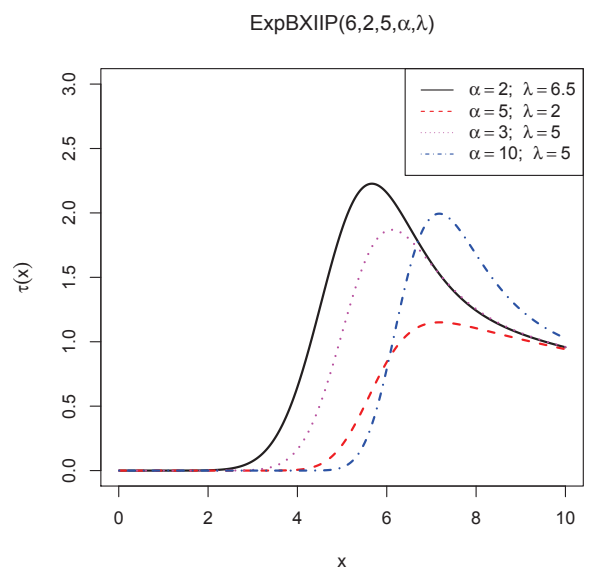

Figure 2. The Exp-BXIIP hrf for some parameter values.

For a second motivation suppose that an ith system is made of $\alpha$ parallel components, so that the system will fail if all of the components fail. Assume that the failure times of the components for the ith system, say $Z_{i, 1}, Z_{i, 2}, \ldots, Z_{i, \alpha}$, are independent and identically BXII random variables with parameters $s, k, c$. Let $Y_{i}$ denote the failure time of the ith system and that there is an unknown number $N$ of independent systems. The cdf of the failure time $X$ of the first system out of the $N$ functioning system is given by (6).

For the third motivation, we assume that $N$ is the unknown number of carcinogenic cells for an individual left active after the initial treatment has pmf (5) and that $Y_{i}$ is the time spent for the $i$ th carcinogenic cell to produce a detectable 
cancer mass. Assuming that $Y_{1}, \ldots, Y_{N}$ is a sequence of iid Exp-BXII random variables independent of $N$, the time to relapse of cancer of a susceptible individual can be modeled by the Exp-BXIIP family of distributions.

Finally, the fourth motivation considers that the failure of a device occurs due to the presence of an unknown number $N$ of initial defects of the same kind, which can be identifiable only after causing failure and are repaired perfectly. Define by $Y_{i}$ the time to the failure of the device due to the $i$ th defect, for $i \geq 1$. If we assume that the $Y_{i}$ 's are iid Exp-BXII random variables independent of $N$ having pmf (5), then the time to the first failure is appropriately modeled by the Exp-BXIIP distribution. For reliability studies, the Exp-BXIIP models can arise in series and parallel systems with identical components, which appear in many industrial applications and biological organisms. These points indicate that the new family of distributions is well-motivated for industrial applications and biological studies.

In this paper, we study some mathematical properties of the Exp-BXIIP model and illustrate its potentiality. In Section 2, we demonstrate that the cdf and pdf of X can be expressed as a mixture of Exp-BXII densities. Explicit expressions for the ordinary and incomplete moments are derived in Section 3. Generating and quantile functions are derived in Section 4 and 5, respectively. In Section 6, mean deviations and reliability are derived. In Section 7, we investigate the order statistics and some of their structural properties. Rényi and Shannon entropies are derived in Section 8. Maximum likelihood estimation of the model parameters is performed and the observed information matrix is determined in Section 9. In Section 10, we provide an application of the Exp-BXIIP to a real data set. Finally, Section 11 ends with some concluding remarks.

\section{Useful Expansions}

Using the Taylor series

$$
1-\mathrm{e}^{-\mathrm{z}}=\sum_{\mathrm{k}=1}^{\infty} \frac{(-1)^{\mathrm{k}+1} \mathrm{z}^{\mathrm{k}}}{\mathrm{k} !}
$$

equation (6) can be expressed as

$$
F(x)=\frac{1}{\left(1-\mathrm{e}^{-\lambda}\right)} \sum_{j=0}^{\infty} \frac{(-1)^{j} \lambda^{j+1}}{(j+1) !}\left\{1-\left[1+\left(\frac{x}{s}\right)^{c}\right]^{-k}\right\}^{(j+1) \alpha},
$$

and then

$$
F(x)=\sum_{j=0}^{\infty} \omega_{j} H_{(j+1) \alpha}(x ; s, k, c)
$$

where $\omega_{j}=\frac{(-1)^{j} \lambda^{j+1}}{(j+1) !\left(1-\mathrm{e}^{-\lambda}\right)}$ and $H_{\alpha}(x ; s, k, c)=G^{\alpha}(x ; s, k, c)$ is the Exp-BXII cdf. Clearly, $\sum_{j=1}^{\infty} w_{j}=1$.

By differentiating (12), we can write

$$
f(x)=\sum_{j=0}^{\infty} \omega_{j} h_{(j+1) \alpha}(x ; s, k, c),
$$

where $h_{(j+1) \alpha}(x ; s, k, c)$ denotes the Exp-BXII fdp with parameters $s, k, c$ and power parameter $(j+1) \alpha$. Equation (13) reveals that the Exp-BXIIP density function is a mixture of Exp-BXII densities.

\section{Properties}

Some of the most important features and characteristics of a distribution can be studied through moments (e.g., tendency, dispersion, skewness and kurtosis).

Theorem 1 If $X \sim \operatorname{Exp}-B X I I P(s, k, c, \alpha, \lambda)$, we have the following approximations:

1.1 For $\alpha>0$ and $\lambda>0$ real non-integers, we have the mixture representation

$$
f(x)=\sum_{r=0}^{\infty} v_{r} g(x ; s, k(r+1), c),
$$

where $g(x ; s, k(r+1), c)$ denotes the BXII density function with scale parameter $s$ and shape parameters $c$ and $k(r+1)$, and the coefficients are given by

$$
v_{r}=\frac{\alpha \lambda}{(r+1) !\left(1-\mathrm{e}^{-\lambda}\right)} \sum_{j=0}^{\infty} \frac{(-1)^{j+r} \lambda^{j} \Gamma[(j+1) \alpha]}{j ! \Gamma[(j+1) \alpha-r]} .
$$


Clearly, $\sum_{r=0}^{\infty} v_{r}=1$. Equation (14) reveals that the Exp-BXIIP density function is an infinite linear combination of BXII density functions. So, some structural properties of the Exp-BXIIP distribution can be obtained from those of the BXII distribution.

1.2 For $\alpha>0$ and $\lambda>0$ real non-integers, we obtain

$$
F(x)=\sum_{r=0}^{\infty} v_{r} G(x ; s, k(r+1), c) .
$$

1.3 If $n<k c$, the nth ordinary moment of the Exp-BXIIP distribution is given by

$$
\mu_{n}^{\prime}=E\left(X^{n}\right)=k s^{n} \sum_{r=0}^{\infty} v_{r} B\left[k(r+1)-\frac{n}{c}, \frac{n}{c}+1\right] .
$$

Proof 1.1.

First, if $z \in \mathbb{R}$, we have the power series

$$
e^{-z}=\sum_{j=0}^{\infty} \frac{(-1)^{j}}{j !} z^{j}
$$

Second, if $|z|<1$ and $b$ is a nonnegative integer, the power series holds

$$
(1-z)^{b-1}=\sum_{j=0}^{\infty} \frac{(-1)^{j} \Gamma(b)}{\Gamma(b-j) j !} z^{j} .
$$

Using (18), the Exp-BXIIP density function (9) can be expressed as

$$
\begin{aligned}
f(x) & =\frac{c k s^{-c} \alpha \lambda}{\left(1-\mathrm{e}^{-\lambda}\right)} x^{c-1}\left[1+\left(\frac{x}{s}\right)^{c}\right]^{-k-1} \sum_{j=0}^{\infty}\left[\frac{(-1)^{j} \lambda^{j}}{j !}\right. \\
& \left.\times\left\{1-\left[1+\left(\frac{x}{s}\right)^{c}\right]^{-k}\right\}^{(j+1) \alpha-1}\right] .
\end{aligned}
$$

Further, using (19), we obtain

$$
\begin{aligned}
f(x) & =\frac{c k(r+1) s^{-c} \alpha \lambda}{\left(1-\mathrm{e}^{-\lambda}\right)} x^{c-1} \sum_{j, r=0}^{\infty}\left\{\frac{(-1)^{j+r} \lambda^{j} \Gamma[(j+1) \alpha]}{j !(r+1) ! \Gamma[(j+1) \alpha-r]}\right. \\
& \left.\times\left[1+\left(\frac{x}{s}\right)^{c}\right]^{-k(r+1)-1}\right\} .
\end{aligned}
$$

Finally, we have

$$
f(x)=\sum_{r=0}^{\infty} v_{r} g(x ; s, k(r+1), c),
$$

where $v_{r}$ is given by (15) and $g(x ; s, k(r+1), c)$ was defined before.

Proof 1.2.

Using Theorem 1.1 we obtain (16) by simple integration.

Proof 1.3.

The $n$th moment of $X$ comes from Theorem 1.1

$$
\mu_{n}^{\prime}=\sum_{r=0}^{\infty} v_{r} E\left(Y_{r+1}\right)
$$


where $Y_{r+1} \sim \operatorname{BXII}(s, k(r+1), c)$. Using a result in Zimmer et al. (1998), we obtain for $n<k c$

$$
\mu_{n}^{\prime}=k s^{n} \sum_{r=0}^{\infty} v_{r} B\left[k(r+1)-n c^{-1}, n c^{-1}+1\right] .
$$

The central moments $\left(\mu_{s}\right)$ and cumulants $\left(\kappa_{s}\right)$ of $X$ can be determined from (17) as

$$
\mu_{s}=\sum_{k=0}^{p}(-1)^{k}\left(\begin{array}{l}
s \\
k
\end{array}\right) \mu_{1}^{\prime s} \mu_{s-k}^{\prime} \quad \text { and } \quad \kappa_{s}=\mu_{s}^{\prime}-\sum_{k=1}^{s-1}\left(\begin{array}{l}
s-1 \\
k-1
\end{array}\right) \kappa_{k} \mu_{s-k}^{\prime},
$$

respectively, where $\kappa_{1}=\mu_{1}^{\prime}$.

For lifetime models, it is usually of interest to compute the $n$th incomplete moment of $X$ defined by $m_{n}(y)=$ $\int_{0}^{y} x^{n} f(x) d x$. The quantity $m_{n}(y)$ can be calculated from (14) as

$$
m_{n}(y)=k c \sum_{r=0}^{\infty}(r+1) v_{r} \int_{0}^{y} x^{n-1}\left(\frac{x}{s}\right)^{c}\left[1+\left(\frac{x}{s}\right)^{-k(r+1)-1}\right] d x .
$$

Setting $t=\left[1+\left(\frac{x}{s}\right)^{c}\right]^{-1}$, we can write

$$
m_{n}(y)=k s^{n} \sum_{r=0}^{\infty}(r+1) v_{r} \int_{0}^{\frac{s^{c}}{s^{c}+y^{c}}} t^{k(r+1)-\frac{n}{c}-1}(1-t)^{\frac{n}{c}} d t
$$

and then for $n<k c$

$$
m_{n}(y)=k s^{n} \sum_{r=0}^{\infty}(r+1) v_{r} B_{\frac{s^{c}}{s^{c}+y^{c}}}\left(k(r+1)-n c^{-1}, 1+n c^{-1}\right),
$$

where $B_{z}(a, b)=\int_{0}^{z} t^{a-1}(1-t)^{b-1} d t$ is the incomplete beta function.

\section{Moment Generating Function}

An explicit expression for $M(t)$ can be obtained from equation (14) as an infinite weighted sum

$$
M(t)=\sum_{r=0}^{\infty} v_{r} M_{r+1}(t)
$$

where $M_{r+1}(t)$ is the moment generating function (mgf) of $Y_{r+1}$ and $v_{r}$ is defined by (15). We provide a simple representation for the $\operatorname{mgf} M_{\mathrm{BXII}}(t)$ of the $\mathrm{BXII}(s, k, c)$ distribution. We can write for $t<0$

$$
M_{\mathrm{BXII}}(t)=c k \int_{0}^{\infty} \mathrm{e}^{\mathrm{yt}} \mathrm{y}^{\mathrm{c}-1}\left(1+\mathrm{y}^{\mathrm{c}}\right)^{-(\mathrm{k}+1)} \mathrm{dy} .
$$

Now, we use the Meijer G-function defined by

$$
G_{p, q}^{m, n}\left(x \mid \begin{array}{l}
a_{1}, \ldots, a_{p} \\
b_{1}, \ldots, b_{q}
\end{array}\right)=\frac{1}{2 \pi i} \int_{L} \frac{\prod_{j=1}^{m} \Gamma\left(b_{j}+t\right) \prod_{j=1}^{n} \Gamma\left(1-a_{j}-t\right)}{\prod_{j=n+1}^{p} \Gamma\left(a_{j}+t\right) \prod_{j=m+1}^{p} \Gamma\left(1-b_{j}-t\right)} x^{-t} d t,
$$

where $\mathrm{i}=\sqrt{-1}$ is the complex unit and L denotes an integration path; see Section 9.3 in Gradshteyn and Ryzhik (2000) for a description of this path. The Meijer G-function contains as particular cases many integrals with elementary and special functions (Prudnikov et al., 1986).

We now assume that $c=m / k$, where $m$ and $k$ are positive integers. This condition is not restrictive since every positive real number can be approximated by a rational number. Using the integral (38) given in Appendix A, we conclude for $t<0$ that

$$
M_{\mathrm{BXII}}(t)=m I\left(-s t, \frac{m}{k}-1, \frac{m}{k},-k-1\right) .
$$


Now, from equation (25), the $\operatorname{mgf}$ of the $\operatorname{Exp}-\operatorname{BXIIP}(s, k, c, \alpha, \lambda)$ distribution (for $t<0$ ) follows as

$$
M(t)=m \sum_{r=0}^{\infty} v_{r} I\left(-s t, \frac{m}{k(r+1)}-1, \frac{m}{k(r+1)},-k(r+1)-1\right) .
$$

Equation (27) is the main result of this section. For the special cases $c=1$ and $c=2$, we can obtain simple expressions for $M_{\mathrm{BXII}}(t)$ and, consequently, for $M(t)$ using equations (1) (on page 16) and (2) (on page 20) of the book by Prudnikov et al. (1992). For $c=1$ and $t<0$, we have

$$
M_{\mathrm{BXII}}(t)=k(-s t)^{k} \mathrm{e}^{-\mathrm{st}} \Gamma(-\mathrm{k},-\mathrm{st}),
$$

where $\Gamma(v, x)=\int_{x}^{\infty} t^{v-1} \mathrm{e}^{-\mathrm{st}} \mathrm{dt}$ is the complementary incomplete gamma function. For $c=2$ and $t<0$, we obtain

$$
\begin{aligned}
M_{\mathrm{BXII}}(t) & ={ }_{1} F_{2}\left(1 ; \frac{1}{2} ; 1-k ; \frac{s^{2} t^{2}}{4}\right)+\frac{s t}{2} B\left(2, k-\frac{1}{2}\right){ }_{1} F_{2}\left(1 ; \frac{3}{2} ; k+\frac{7}{2} ; \frac{-s^{2} t^{2}}{4}\right) \\
& +\frac{\Gamma(-2 k)}{(-s t)^{-2 k}}
\end{aligned}
$$

where

$$
{ }_{1} F_{2}(a, b ; c ; x)=\sum_{k=0}^{\infty} \frac{(a)_{k}}{(b)_{k}(c)_{k}} \frac{x^{k}}{k !}
$$

is a generalized hypergeometric function and $(a)_{k}=a(a+1) \ldots(a+k-1)$ denotes the ascending factorial.

\section{Quantile Function}

The Exp-BXIIP quantile function, say $x=Q(u)$, can be obtained by inverting (6). We have

$$
x=Q(u)=F^{-1}(u)=s\left\{\left[1-\left\{-\lambda^{-1} \log \left[1-u\left(1-\mathrm{e}^{-\lambda}\right)\right]\right\}^{\frac{1}{\alpha}}\right]^{\frac{-1}{k}}-1\right\}^{\frac{1}{c}} .
$$

The shortcomings of the classical kurtosis measure are well-known. For example, the moments of $X$ in (9) are valid only for $n<k c$. There are many heavy-tailed distributions for which this quantile is infinite. So, it becomes uniformative precisely when it needs to be. Indeed, our motivation to use quantile-based measures stemmed from the non-existence of classical kurtosis for many generalized distributions. The Bowley skewness (see Kenney and Keeping, 1962) is based on quartiles

$$
B=\frac{Q(3 / 4)-2 Q(1 / 2)+Q(1 / 4)}{Q(3 / 4)-Q(1 / 4)}
$$

whereas the Moors kurtosis (see Moors, 1998) is based on octiles

$$
M=\frac{Q(7 / 8)-Q(5 / 8)+Q(3 / 8)-Q(1 / 8)}{Q(6 / 8)-Q(2 / 8)},
$$

where $Q(\cdot)$ denotes the Exp-BXIIP quantile function given by (28). Plots of the B and M functions for selected parameter values are displayed in Figure 3.

\section{Other Measures}

In this section, we calculate the following measures: means deviations, Bonferroni and Lorenz curves and the reliability of the Exp-BXIIP distribution.

\subsection{Mean Deviations}

Here, we determine the mean deviations and Bonferroni and Lorenz curves of $X$. The amount of scatter in a population is evidently measured to some extent by the totality of deviations from the mean and median. These are known as the mean deviation about the mean and the mean deviation about the median - defined by

$$
\delta_{1}(X)=2 \mu_{1}^{\prime} F\left(\mu_{1}^{\prime}\right)-2 m_{1}\left(\mu_{1}^{\prime}\right) \quad \text { and } \quad \delta_{2}(X)=\mu_{1}^{\prime}-2 m_{1}(M),
$$

respectively, where $\mu_{1}^{\prime}=E(X), F\left(\mu_{1}^{\prime}\right)$ is obtained from (6), the median $M$ of $X$ is calculated from the quantile function (28) by $M=Q(1 / 2)$ and $m_{1}(q)=\int_{0}^{q} x f(x) d x$ is the incomplete mean of $X$ given by (24) with $n=1$. 


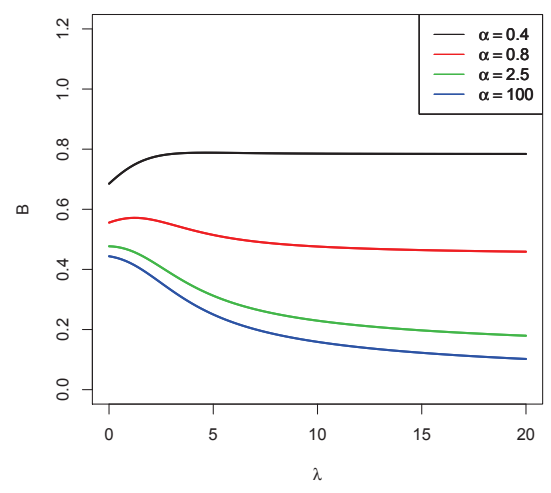

(a) The B function for $s=1.2, k=1.4$ and $c=0.8$.

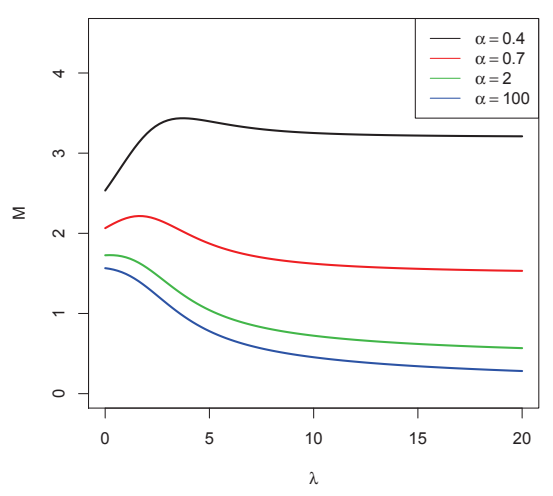

(b) The $\mathrm{M}$ function for $s=1.2, k=1.4$ and $c=0.8$.

Figure 3. Plots of the B and $\mathrm{M}$ functions for some parameter values

Setting $u=y^{c}$, we can write from equation (14)

$$
m_{1}(q)=k s \sum_{r=0}^{\infty}(r+1) v_{r} \int_{(q / s)^{c}}^{\infty} u^{1 / c}(1+u)^{-k(r+1)-1} d u,
$$

where the integral can be calculated using Maple as

$$
m_{1}(q)=k s \sum_{r=0}^{\infty}(r+1) v_{r} J\left(\left[\frac{q}{s}\right]^{c}, \frac{1}{c}, k(r+1)+1\right) .
$$

Here,

$$
\begin{aligned}
J(q, r, k) & =\int_{q}^{\infty} u^{r}(1+u)^{-k} d u \\
& =-\left[\frac{{ }_{2} F_{1}[(k, r+1) ;(2+r) ;-q] q^{r+1}}{(r+1)}+\frac{\pi \Gamma(k-r-1) \csc (\pi r)}{\Gamma(k) \Gamma(-r)}\right],
\end{aligned}
$$

where $\csc (\cdot)$ is the $\operatorname{cosecant}$ function and ${ }_{2} F_{1}$ is the hypergeometric function defined by

$$
{ }_{2} F_{1}(a, b ; c ; x)=\sum_{k=0}^{\infty} \frac{(a)_{k}(b)_{k}}{(c)_{k}} \frac{x^{k}}{k !} .
$$

Equation (29) is the main result of this section from which $\delta_{1}(X)$ and $\delta_{2}(X)$ are immediately determined. The mean deviations can be used to plot Lorenz and Bonferroni curves in fields like economics, reliability, demography, insurance and medicine. For a given probability $\pi$, they are defined by $L(\pi)=m_{1}(q) / \mu_{1}^{\prime}$ and $B(\pi)=m_{1}(q) /\left(\pi \mu_{1}^{\prime}\right)$, respectively, where $q=Q(\pi)$ comes directly from (28).

\subsection{Reliability}

In reliability, the stress-strength model describes the life of a component which has a random strength $X_{1}$ that is subjected to a random stress $X_{2}$. The component fails at the instant that the stress applied to it exceeds the strength, and the component will function satisfactorily whenever $X_{1}>X_{2}$. Hence, $R=\operatorname{Pr}\left(X_{2}<X_{1}\right)$ is a measure of component reliability. It has many applications especially in engineering concepts, economics and physical science. We derive the reliability $R$ when $X_{1}$ and $X_{2}$ have independent $\operatorname{Exp}-\operatorname{BXIIP}\left(s, k_{1}, c, \alpha_{1}, \lambda_{1}\right)$ and $\operatorname{Exp}-\operatorname{BXIIP}\left(s, k_{2}, c, \alpha_{2}, \lambda_{2}\right)$ distributions with identical scale parameter $s$ and shape parameter $c$. The reliability is given by

$$
R=\int_{0}^{\infty} f_{1}(x) F_{2}(x) d x
$$


The cdf of $X_{2}$ and density of $X_{1}$ are obtained from Theorem 1

$$
F_{2}(x)=\sum_{r=0}^{\infty} v_{r}\left(\alpha_{2}, \lambda_{2}\right) G\left(x ; s, k_{2}(r+1), c\right)
$$

and

$$
f_{1}(x)=\sum_{q=0}^{\infty} v_{q}\left(\alpha_{1}, \lambda_{1}\right) g\left(x ; s, k_{1}(q+1), c\right) .
$$

Hence,

$$
R=\frac{c k_{1}}{s^{c}} \sum_{r, q=0}^{\infty}(q+1) v_{q}\left(\alpha_{1}, \lambda_{1}\right) v_{r}\left(\alpha_{2}, \lambda_{2}\right) I\left(c, s, k_{1}, k_{2}, r, q\right)
$$

where

$$
\begin{aligned}
I\left(c, s, k_{1}, k_{2}, r, q\right) & =\int_{0}^{\infty}\left(x^{c-1}\left[1+\left(\frac{x}{s}\right)^{c}\right]^{-k_{1}(q+1)-1}\right. \\
& \left.\times\left\{1-\left[1+\left(\frac{x}{s}\right)^{c}\right]^{-k_{2}(r+1)}\right\}\right) d x
\end{aligned}
$$

Setting $u=1+(x / s)^{c}$, we have

$$
I\left(c, s, k_{1}, k_{2}, r, q\right)=\frac{c k_{2}(r+1)-1}{c k_{1}(q+1)+c k_{2}(r+1)},
$$

and then we obtain $R$.

\section{Order Statistics}

We now derive an explicit expression for the density of the $i$ th order statistic $X_{i: n}$, say $f_{i: n}(x)$, in a random sample of size $n$ from the Exp-BXIIP distribution. It is well-known that

$$
f_{i: n}(x)=\frac{1}{B(i, n-i+1)} f(x) F(x)^{i-1}[1-F(x)]^{n-i},
$$

for $i=1, \ldots, n$. Using the binomial expansion in the last equation, we readily obtain

$$
f_{i: n}(x)=\sum_{l=0}^{n-i} \frac{(-1)^{l}\left(\begin{array}{c}
n-i \\
l
\end{array}\right) f(x)}{B(i, n-i+1)} F(x)^{i+l-1} .
$$

We use the identity for $k, v$ positive integer

$$
\left(\sum_{k=0}^{\infty} a_{k} x^{k}\right)^{v}=\sum_{k=0}^{\infty} c_{v, k} x^{k}
$$

where $c_{v, 0}=a_{0}^{v}$ and

$$
c_{v, j}=\frac{1}{j a_{0}} \sum_{q=1}^{j}\left[(v q-j+q) a_{q} c_{v, j-q}\right] .
$$

Then, we can write

$$
\begin{aligned}
F^{v}(x) & =\frac{1}{\left(1-e^{-\lambda}\right)^{v}}\left\{1-\left[1+\left(\frac{x}{s}\right)^{c}\right]^{-k}\right\}^{v \alpha}\left\{\sum_{j=0}^{\infty} \frac{(-1)^{j} \lambda^{j+1}}{(j+1) !}\right. \\
& \left.\times\left\{1-\left[1+\left(\frac{x}{s}\right)^{c}\right]^{-k}\right\}^{j \alpha}\right\} \\
& =\frac{1}{\left(1-e^{-\lambda}\right)^{v}}\left\{1-\left[1+\left(\frac{x}{s}\right)^{c}\right]^{-k}\right\}^{v \alpha} \sum_{j=0}^{\infty} c_{v, j}\left\{1-\left[1+\left(\frac{x}{s}\right)^{c}\right]^{-k}\right\}^{j \alpha} \\
& =\frac{1}{\left(1-e^{-\lambda}\right)^{v}} \sum_{j=0}^{\infty} c_{v, j}\left\{1-\left[1+\left(\frac{x}{s}\right)^{c}\right]^{-k}\right\}^{(j+v) \alpha}
\end{aligned}
$$


where $c_{v, j}$ is given in (32) and $a_{j}=\frac{(-1)^{j} \lambda^{j+1}}{(j+1) !}$.

Setting $v=i+l-1$ and substituting (20) and (33) into equation (30), the density $f_{i: n}(x)$ can be expressed as

$$
\begin{aligned}
f_{i: n}(x) & =\frac{1}{\left(1-e^{-\lambda}\right)^{i+l-1}} \sum_{l=0}^{n-i} \sum_{j=0}^{\infty}\left[\frac{(-1)^{l}\left(\begin{array}{c}
n-i \\
l
\end{array}\right) c_{i+l-1, j} f(x)}{B(i, n-i+1)}\right. \\
& \left.\times\left\{1-\left[1+\left(\frac{x}{s}\right)^{c}\right]^{-k}\right\}\right]^{(i+j+l-1) \alpha} \\
& =\frac{1}{\left(1-e^{-\lambda}\right)^{i+l}} \sum_{l=0}^{n-i} \sum_{j, m=0}^{\infty} \frac{(-1)^{l+m}\left(\begin{array}{c}
n-i \\
l
\end{array}\right) \lambda^{m} c_{i+l-1, j}}{m ! B(i, n-i+1)} c k s^{-c} \alpha \lambda x^{c-1} \\
& \times\left[1+\left(\frac{x}{s}\right)^{c}\right]^{-k-1}\left\{1-\left[1+\left(\frac{x}{s}\right)^{c}\right]^{-k}\right\}^{(i+j+l+m) \alpha-1} \\
& =\frac{1}{\left(1-e^{-\lambda}\right)^{i+l}} \sum_{l=0}^{n-i} \sum_{j, m=0}^{\infty} \frac{(-1)^{l+m}\left(\begin{array}{c}
n-i \\
l
\end{array}\right) \lambda^{m+1} c_{i+l-1, j}}{(i+j+l+m) m ! B(i, n-i+1)} c k s^{-c} \alpha \\
& \times(i+j+l+m) x^{c-1}\left[1+\left(\frac{x}{s}\right)^{c}\right]^{-k-1}\left\{1-\left[1+\left(\frac{x}{s}\right)^{c}\right]^{-k}\right\}^{(i+j+l+m) \alpha-1} .
\end{aligned}
$$

Thus, $f_{i: n}(x)$ can be written as

$$
f_{i: n}(x)=\sum_{l=0}^{n-i} \sum_{j, m=0}^{\infty} \delta_{j, l, m} h_{(i+j+l+m) \alpha}(x),
$$

where

$$
\delta_{j, l, m}=\frac{(-1)^{l+m\left(\begin{array}{c}
n-i \\
l
\end{array}\right) \lambda^{m+1} c_{i+l-1, j}}}{(i+j+l+m) m !\left(1-\mathrm{e}^{-\lambda}\right)^{\mathrm{i}+1} \mathrm{~B}(\mathrm{i}, \mathrm{n}-\mathrm{i}+1)},
$$

and

$$
\begin{aligned}
h_{(i+j+l+m) \alpha}(x) & =c k s^{-c}(i+j+l+m) \alpha x^{c-1}\left[1+\left(\frac{x}{s}\right)^{c}\right]^{-k-1} \\
& \times\left\{1-\left[1+\left(\frac{x}{s}\right)^{c}\right]^{-k}\right\}^{(i+j+l+m) \alpha-1}
\end{aligned}
$$

Thus, from equation (34), the $t$ th ordinary moment of the Exp-BXIIP order statistics is

$$
\mathrm{E}\left(X_{i: n}^{t}\right)=\sum_{l=0}^{n-i} \sum_{j, m=0}^{\infty} \delta_{j, l, m} \mathrm{E}\left(Y_{(i+j+l+m) \alpha}\right),
$$

where $Y_{(i+j+l+m) \alpha} \sim \operatorname{Exp}-\mathrm{BXII}\left(s, k, c, Y_{(i+j+l+m) \alpha}\right)$. Clearly, $\mathrm{E}\left(X_{i, l}^{t}\right)$ can be calculated directly from equation (23) with the parameters of this Exp-BXII distribution.

An alternative expression to (35) can be derived using a result due to Barakat and Abdelkader (2004). We have

$$
\mathrm{E}\left(X_{i: n}^{t}\right)=t \sum_{p=n-i+1}^{n}(-1)^{p-n+i-1}\left(\begin{array}{l}
p-1 \\
n-i
\end{array}\right)\left(\begin{array}{l}
n \\
p
\end{array}\right) \int_{0}^{\infty} x^{t-1} S(x)^{p} d x,
$$

where $S(x)=1-F(x)$ is the Exp-BXIIP survival function. Using the binomial expansion for $[1-F(x)]^{p}$ in (36), the last integral becomes

$$
L=\int_{0}^{\infty} x^{t-1} S(x)^{p} d x=\sum_{l=0}^{p}(-1)^{l}\left(\begin{array}{l}
p \\
l
\end{array}\right) \int_{0}^{\infty} x^{t-1} F(x)^{l} d x
$$


Substituting (33) into equation (37), we can rewrite L as

$$
L=\sum_{l=0}^{p} \sum_{j=0}^{\infty} \frac{(-1)^{l}\left(\begin{array}{l}
p \\
l
\end{array}\right) c_{l, j}}{\left(1-\mathrm{e}^{-\lambda}\right)^{1}} \int_{0}^{\infty} x^{t-1}\left\{1-\left[1+\left(\frac{x}{s}\right)^{c}\right]^{-k}\right\}^{(j+l) \alpha} d x
$$

where $c_{l, j}$ can be obtained from (32).

Using the power series expansion, we can write $L$ as

$$
L=\sum_{l=0}^{p} \sum_{j, m=0}^{\infty} \frac{(-1)^{l+m}\left(\begin{array}{l}
p \\
l
\end{array}\right) c_{l, j} \Gamma[(j+l) \alpha+1]}{m !\left(1-\mathrm{e}^{-\lambda}\right)^{1} \Gamma[(\mathrm{j}+\mathrm{l}) \alpha+1-\mathrm{m}]} \int_{0}^{\infty} x^{t-1}\left[1+\left(\frac{x}{s}\right)^{c}\right]^{-k m} d x
$$

Setting $u=(x / s)^{c}$, we obtain

$$
L=\sum_{l=0}^{p} \sum_{j, m=0}^{\infty} \frac{(-1)^{l+m}\left(\begin{array}{l}
p \\
l
\end{array}\right) s^{t} c_{l, j} \Gamma[(j+l) \alpha+1]}{c m !\left(1-\mathrm{e}^{-\lambda}\right)^{1} \Gamma[(\mathrm{j}+1) \alpha+1-\mathrm{m}]}\left[\frac{\Gamma\left(k m-\frac{t}{c}\right) \Gamma\left(\frac{t}{c}\right)}{\Gamma(k m)}\right] .
$$

Finally, equation (36) reduces to

$$
\begin{aligned}
\mathrm{E}\left(X_{i: n}^{t}\right) & =t \sum_{p=n-i+1}^{n}(-1)^{p-n+i-1}\left(\begin{array}{c}
p-1 \\
n-i
\end{array}\right)\left(\begin{array}{l}
n \\
p
\end{array}\right) \sum_{l=0}^{p} \sum_{j, m=0}^{\infty} \frac{(-1)^{l+m}\left(\begin{array}{l}
p \\
l
\end{array}\right) s^{t} c_{l, j}}{c m !\left(1-\mathrm{e}^{-\lambda}\right)^{1}} \\
& \times\left[\frac{\Gamma[(j+l) \alpha+1] \Gamma\left(k m-\frac{t}{c}\right) \Gamma\left(\frac{t}{c}\right)}{\Gamma[(j+l) \alpha+1-m] \Gamma(k m)}\right] .
\end{aligned}
$$

The L moments (Hosking, 1990) are expectations of certain linear combinations of order statistics and can be used to calculate quantities analogous to standard deviation, skewness and kurtosis, termed the L scale, L skewness and L kurtosis respectively. They are defined by

$$
\lambda_{r+1}=(r+1)^{-1} \sum_{k=0}^{r}(-1)^{k}\left(\begin{array}{l}
r \\
k
\end{array}\right) E\left(X_{r+1-k: r+1}\right), r=0,1, \ldots
$$

The first four L-moments are: $\lambda_{1}=E\left(X_{1: 1}\right), \lambda_{2}=\frac{1}{2} E\left(X_{2: 2}-X_{1: 2}\right), \lambda_{3}=\frac{1}{3} E\left(X_{3: 3}-2 X_{2: 3}+X_{1: 3}\right)$ and $\lambda_{4}=\frac{1}{4} E\left(X_{4: 4}-\right.$ $\left.3 X_{3: 4}+3 X_{2: 4}-X_{1: 4}\right)$. From equation (35) for the moments of the order statistics, we can obtain expansions for the L-moments of the Exp-BXIIP distribution as linear functions of the means of suitable Exp-BXII distributions.

\section{Rényi and Shannon Entropy}

The entropy of a random variable $X$ with density function $f(x)$ is a measure of variation of the uncertainty. For any real parameter $\omega>0$ and $\omega \neq 1$, the Rényi entropy of the Exp-BXIIP distribution is given by

$$
\begin{aligned}
I_{R}(\gamma) & =\frac{1}{(1-\gamma)} \log \int_{0}^{\infty} f^{\gamma}(x) d x \\
& =\frac{1}{(1-\gamma)} \log \left\{\left[\frac{c k s^{-c} \alpha \lambda}{1-\mathrm{e}^{-\lambda}}\right]^{\gamma} \sum_{j, r=0}^{\infty} \frac{(-1)^{j+r} \lambda^{j} \gamma^{j}\left(\begin{array}{c}
(j+r) \alpha-\gamma \\
r
\end{array}\right)}{j !}\right. \\
& \left.\times \int_{0}^{\infty} x^{(c-1) \gamma}\left[1+\left(\frac{x}{s}\right)^{c}\right]^{-k(r+\gamma)-\gamma} d x\right\} \\
& =\frac{1}{(1-\gamma)} \log \left\{\left[\frac{c k s^{-c} \alpha \lambda}{1-\mathrm{e}^{-\lambda}}\right]^{\gamma} \frac{s^{(c-1) \gamma+1}}{c} \sum_{j, r=0}^{\infty} \frac{(-1)^{j+r} \lambda^{j} \gamma^{j}\left(\begin{array}{c}
(j+r) \alpha-\gamma \\
r
\end{array}\right)}{j !}\right. \\
& \left.\times\left[\frac{\Gamma\left[\frac{(c-1) \gamma+1}{c}\right] \Gamma\left[\frac{c k(r+\gamma)+\gamma-1}{c}\right]}{\Gamma[k(r+\gamma)+\gamma]}\right]\right\} .
\end{aligned}
$$

The details of the proof are given in Appendix B. 
For the Shannon entropy, we have

$$
\begin{aligned}
E\{-\log [f(X)]\} & =\log \left(1-\mathrm{e}^{-\lambda}\right)-\log (\alpha)-\log (\lambda) \\
& +(\alpha-1) \sum_{j, r=0}^{\infty} \frac{(r+1) v_{r}}{(j+1)(j+r+2)} \\
& +\lambda \sum_{j, r=0}^{\infty} \frac{(-1)^{j}(r+1) v_{r} \Gamma(\alpha+1)}{(j+r+1) j ! \Gamma(\alpha-j)}-E\{\log [g(X ; s, k, c)]\},
\end{aligned}
$$

where $v_{r}$ is defined in Theorem 1 and $E\{\log [g(X ; s, k, c)]\}$ can be computed from (2) at least numerically. The details of the proof are given in Appendix C.

\section{Estimation}

Let $X_{i}$ be a random variable following (9) with the vector $\boldsymbol{\theta}=(s, k, c, \alpha, \lambda)^{T}$ of parameters. The data encountered in survival analysis and reliability studies are often censored. The censored log-likelihood $l(\boldsymbol{\theta})$ for the model parameters is

$$
\begin{aligned}
l(\boldsymbol{\theta}) & =\log (c)+\log (k)-c \log (s)+\log (\alpha)+\log (\lambda)-\log \left(1-\mathrm{e}^{-\lambda}\right) \\
& +\frac{(c-1)}{n} \sum_{i=1}^{n} \log \left(x_{i}\right)+\frac{k}{n} \sum_{i=1}^{n} \log \left(q_{i,-1}\right)+\frac{(\alpha-1)}{n} \sum_{i=1}^{n} \log \left(v_{i, 1}\right) \\
& -\frac{\lambda}{n} \sum_{i=1}^{n} v_{i, \alpha} .
\end{aligned}
$$

The score functions for the parameters $s, k, c \alpha$ and $\lambda$ are given by

$$
\begin{gathered}
U_{s}(\boldsymbol{\theta})=-\frac{c}{s}-\frac{k c}{n s} \sum_{i=1}^{n} u_{i} q_{i, 1}-\frac{c k(\alpha-1)}{n s} \sum_{i=1}^{n} \frac{u_{i} q_{i, k+1}}{v_{i, 1}}, \\
U_{k}(\boldsymbol{\theta})=\frac{1}{k}+\frac{1}{n} \sum_{i=1}^{n} \log \left(q_{i,-1}\right)+\frac{(\alpha-1)}{n} \sum_{i=1}^{n} \frac{q_{i, k} \log \left(q_{i,-1}\right)}{v_{i, 1}}, \\
U_{c}(\boldsymbol{\theta})=\frac{1}{c}-\log (s)+\frac{1}{n} \sum_{i=1}^{n} \log \left(x_{i}\right)+\frac{k}{n} \sum_{i=1}^{n} \frac{u_{i} \log \left(u_{i}^{1 / c}\right)}{q_{i,-1}}, \\
U_{\alpha}(\boldsymbol{\theta})=\frac{1}{\alpha}+\frac{1}{n} \sum_{i=1}^{n} \log \left(v_{i, 1}\right)-\frac{\lambda}{n} \sum_{i=1}^{n} v_{i, \alpha} \log \left(v_{i, 1}\right),
\end{gathered}
$$

and

$$
U_{\lambda}(\boldsymbol{\theta})=\frac{1}{\lambda}-\frac{\mathrm{e}^{-\lambda}}{1-\mathrm{e}^{-\lambda}}-\sum_{i=1}^{n} v_{i, \alpha}
$$

where $u_{i}=\left(\frac{x_{i}}{s}\right)^{c}, q_{i, k}=\left[1+\left(\frac{x_{i}}{s}\right)^{c}\right]^{-k}$ and $v_{i, \alpha}=\left\{1-\left[1+\left(\frac{x_{i}}{s}\right)^{c}\right]^{-k}\right\}^{\alpha}$.

The maximum likelihood estimate (MLE) $\widehat{\boldsymbol{\theta}}$ of $\boldsymbol{\theta}$ is obtained by solving the nonlinear likelihood equations $U_{s}(\boldsymbol{\theta})=$ $0, U_{k}(\boldsymbol{\theta})=0, U_{c}(\boldsymbol{\theta})=0, U_{\alpha}(\boldsymbol{\theta})=0$ and $U_{\lambda}(\boldsymbol{\theta})=0$. These equations cannot be solved analytically and statistical software can be used to solve them numerically. We can use iterative techniques such as a Newton-Raphson type algorithm to obtain $\widehat{\boldsymbol{\theta}}$. The computations are performed using the software R version 3.0.0 (package bbmle).

For interval estimation of $(s, k, c, \alpha, \lambda)$ and hypothesis tests on these parameters, we obtain the observed information matrix since its expectation requires numerical integration. The $5 \times 5$ observed information matrix $J(\theta)$ is

$$
J(\boldsymbol{\theta})=-\left(\begin{array}{ccccc}
\mathbf{U}_{s s} & \mathbf{U}_{s k} & \mathbf{U}_{s c} & \mathbf{U}_{s \alpha} & \mathbf{U}_{s \lambda} \\
\cdot & \mathbf{U}_{k k} & \mathbf{U}_{k c} & \mathbf{U}_{k \alpha} & \mathbf{U}_{k \lambda} \\
\cdot & \cdot & \mathbf{U}_{c c} & \mathbf{U}_{c \alpha} & \mathbf{U}_{c \lambda} \\
\cdot & \cdot & \cdot & \mathbf{U}_{\alpha \alpha} & \mathbf{U}_{\alpha \lambda} \\
\cdot & \cdot & \cdot & & \mathbf{U}_{\lambda \lambda}
\end{array}\right),
$$


whose elements are given in Appendix D. The matrix $J(\theta)$ is useful to obtain approximate confidence intervals for the parameters.

\section{Application}

In this section, we illustrate the usefulness of the Exp-BXIIP distribution applied to a real data set. These data on failure times are reported in the book "Weibull Models by Murthy" et al. (2004, page 297). We also fit the density functions of the Exponentiated Burr XII Poisson (Exp-BXIIP), Beta Burr XII (BBXII), Kumaraswamy Burr XII (KwBXII) and McDonald Burr XII (McBXII) distributions given by

$$
\begin{aligned}
& f_{\text {Exp-BXIIP }}(x ; s, k, c, \alpha, \lambda)=\frac{c k s^{-c} \alpha \lambda}{1-\mathrm{e}^{-\lambda}} x^{c-1}\left[1+\left(\frac{x}{s}\right)^{c}\right]^{-k-1}\left\{1-\left[1+\left(\frac{x}{s}\right)^{c}\right]^{-k}\right\}^{\alpha-1} \\
& \times \exp \left\{-\lambda\left[1-\left(1+\left(\frac{x}{s}\right)^{c}\right)^{-k}\right]^{\alpha}\right\}, \\
& f_{\mathrm{BBXII}}(x ; s, k, c, a, b)=\frac{c k s^{-c}}{B(a, b)} x^{c-1}\left[1+\left(\frac{x}{s}\right)^{c}\right]^{-k b-1}\left\{1-\left[1+\left(\frac{x}{s}\right)^{c}\right]^{-k}\right\}^{a-1}, \\
& f_{\mathrm{KwBXII}}(x ; s, k, c, a, b)=a b c k s^{-c} x^{c-1}\left[1+\left(\frac{x}{s}\right)^{c}\right]^{-k-1}\left\{1-\left[1+\left(\frac{x}{s}\right)^{c}\right]^{-k}\right\}^{\alpha-1} \\
& \times\left\{1-\left\{1-\left[1+\left(\frac{x}{s}\right)^{c}\right]^{-k}\right\}^{a}\right\}^{b-1}, \\
& f_{\text {McBXIIP }}(x ; s, k, c, a, b, \alpha)=\frac{c k s^{-c} \alpha}{B(a, b)} x^{c-1}\left[1+\left(\frac{x}{s}\right)^{c}\right]^{-k-1}\left\{1-\left[1+\left(\frac{x}{s}\right)^{c}\right]^{-k}\right\}^{a \alpha-1} \\
& \times\left\{1-\left\{1-\left[1+\left(\frac{x}{s}\right)^{c}\right]^{-k}\right\}^{\alpha}\right\}^{b-1},
\end{aligned}
$$

respectively, where all parameters are positive.

Further, we apply the Cramér-von Mises $\left(W^{*}\right)$ and Anderson-Darling $\left(A^{*}\right)$ statistics described in details in Chen and Balakrishnan (1995) to verify which distribution fits better to these data. In general, the smaller the values of the statistics $W^{*}$ and $A^{*}$, the better the fit to the data. Let $H(x ; \boldsymbol{\theta})$ be the cdf, where the form of $H$ is known but $\boldsymbol{\theta}$ (a $k$-dimensional parameter vector, say) is unknown. To obtain the statistics $W^{*}$ and $A^{*}$, one can proceed as follows: (i) Compute $v_{i}=H\left(x_{i} ; \widehat{\boldsymbol{\theta}}\right)$, where the $x_{i}$ 's are in ascending order; (ii) Compute $y_{i}=\Phi^{-1}\left(v_{i}\right)$, where $\Phi(\cdot)$ is the standard normal cdf and $\Phi^{-1}(\cdot)$ its inverse; (iii) Compute $u_{i}=\Phi\left\{\left(y_{i}-\bar{y}\right) / s_{y}\right\}$, where $\bar{y}=n^{-1} \sum_{i=1}^{n} y_{i}$ and $s_{y}^{2}=(n-1)^{-1} \sum_{i=1}^{n}\left(y_{i}-\bar{y}\right)^{2}$; (iv) Calculate $W^{2}=\sum_{i=1}^{n}\left\{u_{i}-(2 i-1) /(2 n)\right\}^{2}+1 /(12 n)$ and $A^{2}=-n-(1 / n) \sum_{i=1}^{n}\{(2 i-$

\begin{tabular}{|c|c|c|c|c|c|c|}
\hline Distribution & & & istimative & & & \\
\hline \multirow[t]{3}{*}{ Exp-BXIIP } & $\widehat{s}$ & $\widehat{k}$ & $\widehat{c}$ & $\widehat{\alpha}$ & $\widehat{\lambda}$ & \\
\hline & 14.8518 & 5.9646 & 5.3264 & 0.4471 & 22.7252 & \\
\hline & $(0.0726)$ & $(0.2732)$ & $(0.0734)$ & $(0.0427)$ & $(5.9532)$ & \\
\hline \multirow[t]{3}{*}{ KwBXII } & $\widehat{s}$ & $\widehat{k}$ & $\widehat{c}$ & $\widehat{a}$ & $\widehat{b}$ & \\
\hline & 5.7916 & 6.3749 & 7.0604 & 0.2510 & 1.4676 & \\
\hline & $(1.3907)$ & $(5.8618)$ & $(0.0207)$ & $(0.0584)$ & $(0.9002)$ & \\
\hline \multirow[t]{3}{*}{ McBXII } & $\widehat{s}$ & $\widehat{k}$ & $\widehat{c}$ & $\widehat{a}$ & $\widehat{b}$ & $\widehat{\alpha}$ \\
\hline & 6.5058 & 6.7187 & 6.6588 & 0.5303 & 2.3546 & 0.5081 \\
\hline & $(0.0103)$ & (6.6788) & $(0.0103)$ & $(0.6782)$ & (1.8471) & $(0.6112)$ \\
\hline \multirow[t]{3}{*}{ BBXII } & $\widehat{s}$ & $\widehat{k}$ & $\widehat{c}$ & $\widehat{a}$ & $\widehat{b}$ & \\
\hline & 7.5361 & 6.5139 & 6.3234 & 0.2584 & 6.4360 & \\
\hline & $(0.0553)$ & $(9.2920)$ & $(0.0599)$ & $(0.0357)$ & (9.1064) & \\
\hline
\end{tabular}
1) $\left.\log \left(u_{i}\right)+(2 n+1-2 i) \log \left(1-u_{i}\right)\right\}$; (v) Modify $W^{2}$ into $W^{*}=W^{2}(1+0.5 / n)$ and $A^{2}$ into $A^{*}=A^{2}\left(1+0.75 / n+2.25 / n^{2}\right)$. Table 1 and 2, respectively, lists the MLEs, their standard errors in parentheses and the statistics $W^{*}$ and $A^{*}$ and $p$ values for the failure times data. They indicate that the Exp-BXIIP and McBXII distributions are the best models to these data. Morever, the standard errors are much smaller compared with their estimates for the Exp-BXIIP distribution.

Table 1. MLEs 
Table 2. Measures $W^{*}$ and $A^{*}$

\begin{tabular}{ccccc}
\hline Distribution & $W^{*}$ & $p$-value & $A^{*}$ & $p$-value \\
\hline Exp-BXIIP & 0.05853 & 0.395 & 0.58938 & 0.124 \\
\hline \hline KwBXII & 0.09915 & 0.115 & 0.68478 & 0.074 \\
\hline \hline McBXII & 0.10423 & 0.098 & 0.95368 & 0.016 \\
\hline \hline BBXII & 0.13694 & 0.035 & 1.19636 & 0.004 \\
\hline
\end{tabular}

More information is provided by a visual comparison of the fitted densities to the histogram of the data. The plots of the fitted Exp-BXIIP, BBXII, KwBXII and McBXII density functions are displayed in Figure 4. These plots indicate that the new distribution provides a good fit to these data and that it is also a very compettitive model to other lifetime distributions.

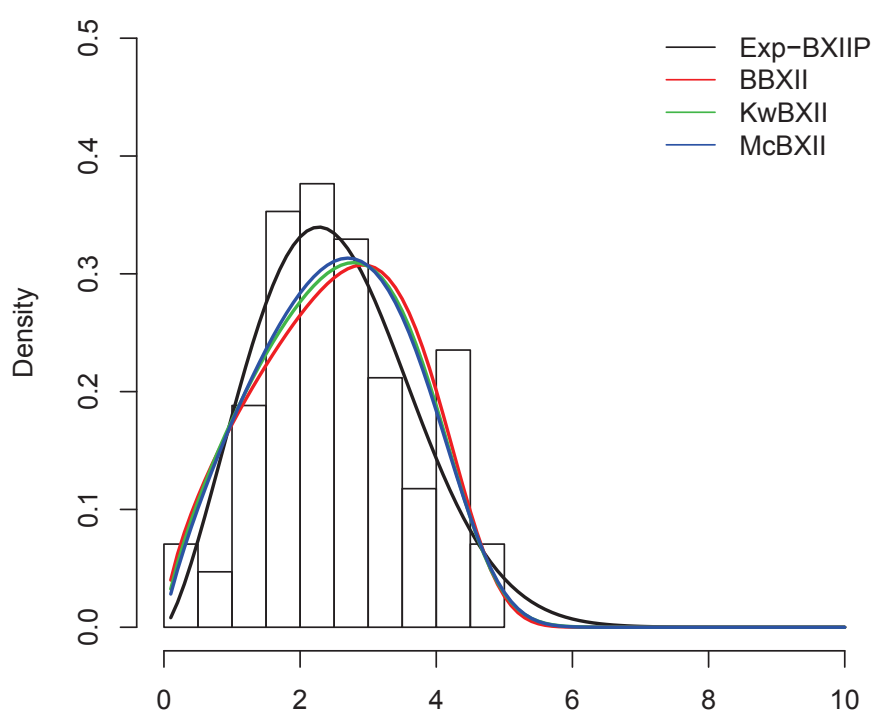

Figure 4. Fitted densities to the histogram of the current data.

\section{Conclusions}

We define and study a new five-parameter lifetime model called the exponentiated Burr XII Poisson distribution, which extends some well-known lifetime distributions. Due to its flexibility in accommodating different forms of the hazard rate function, it is an important model for modeling lifetime data. We provide a mathematical treatment of the proposed distribution including a useful expansion for its density function. We derive explicit expressions for the moments, generating and quantile functions, mean deviations, reliability and entropies, which hold in generality for any parameter values. The model parameters are estimated by maximum likelihood. Additionally, the observed information matrix is determined. In one application to a real data set, we illustrate the potentiality of the new model.

\section{Appendix A - Generating function}

We have the following result which holds for $m$ and $k$ positive integers, $\mu>-1$ and $p>0$ (Prudnikov et al., 1992, 
page 21)

$$
\begin{aligned}
I\left(p, \mu, \frac{m}{k}, v\right)= & \int_{0}^{\infty} \exp (-p x) x^{\mu}\left(1+x^{\frac{m}{k}}\right)^{v} d x \\
= & \frac{k^{-v} m^{\mu+\frac{1}{2}}}{(2 \pi)^{\frac{(m-1)}{2}} \Gamma(-v) p^{\mu+1}} \times \\
& G_{k+m, k}^{k, k+m}\left(\frac{m^{m}}{p^{m}} \mid \begin{array}{l}
\Delta(m,-\mu), \Delta(k, v+1) \\
\Delta(k, 0)
\end{array}\right),
\end{aligned}
$$

where $\Delta(k, a)=\frac{a}{k}, \frac{a+1}{k}, \cdots, \frac{a+k}{k}$.

\section{Appendix B - Rényi entropy}

The entropy of a random variable $X$ with density function $f(x)$ is a measure of variation of the uncertainty. For any real parameter $\omega>0$ and $\omega \neq 1$, the Rényi entropy is given by

$$
I_{R}(\gamma)=\frac{1}{(1-\gamma)} \log \int_{0}^{\infty} f^{\gamma}(x) d x
$$

where

$$
\begin{aligned}
f(x)^{\gamma} & =\left[\frac{c k s^{-c} \alpha \lambda}{1-\mathrm{e}^{-\lambda}}\right]^{\gamma} x^{(c-1) \gamma}\left[1+\left(\frac{x}{s}\right)^{c}\right]^{-(k+1) \gamma}\left\{1-\left[1+\left(\frac{x}{s}\right)^{c}\right]^{-k}\right\}^{(\alpha-1) \gamma} \\
& \times \exp \left\{-\lambda \gamma\left\{1-\left[1+\left(\frac{x}{s}\right)^{c}\right]^{-k}\right\}^{\alpha}\right\} \\
& =\left[\frac{c k s^{-c} \alpha \lambda}{1-\mathrm{e}^{-\lambda}}\right]^{\gamma} x^{(c-1) \gamma}\left[1+\left(\frac{x}{s}\right)^{c}\right]^{-(k+1) \gamma} \sum_{j=0}^{\infty} \frac{(-1)^{j} \lambda^{j} \gamma^{j}}{j !} \\
& \times\left\{1-\left[1+\left(\frac{x}{s}\right)^{c}\right]^{-k}\right\} \\
& =\left[\frac{c k s^{-c} \alpha \lambda}{1-\mathrm{e}^{-\lambda}}\right]^{\gamma} x^{(c-1) \gamma} \sum_{j, r=0}^{\infty} \frac{(-1)^{j+r} \lambda^{j} \gamma^{j}((j+\gamma) \alpha-\gamma)}{j !}\left[1+\left(\frac{x}{s}\right)^{c}\right]^{-k(r+\gamma)-\gamma} .
\end{aligned}
$$

Thus,

$$
\begin{aligned}
I_{R}(\gamma)= & \frac{1}{(1-\gamma)} \log \left\{\left[\frac{c k s^{-c} \alpha \lambda}{1-\mathrm{e}^{-\lambda}}\right]^{\gamma} \sum_{j, r=0}^{\infty} \frac{(-1)^{j+r} \lambda^{j} \gamma^{j}\left(\begin{array}{l}
(j+r) \alpha-\gamma \\
r
\end{array}\right)}{j !}\right. \\
& \left.\times \int_{0}^{\infty} x^{(c-1) \gamma}\left[1+\left(\frac{x}{s}\right)^{c}\right]^{-k(r+\gamma)-\gamma} d x\right\} \\
= & \frac{1}{(1-\gamma)} \log \left\{\left[\frac{c k s^{-c} \alpha \lambda}{1-\mathrm{e}^{-\lambda}}\right]^{\gamma} \frac{s^{(c-1) \gamma+1}}{c} \sum_{j, r=0}^{\infty} \frac{(-1)^{j+r} \lambda^{j} \gamma^{j}\left(\begin{array}{c}
(j+r) \alpha-\gamma \\
r
\end{array}\right)}{j !}\right. \\
& \left.\times\left[\frac{\Gamma\left[\frac{(c-1) \gamma+1}{c}\right] \Gamma\left[\frac{c k(r+\gamma)+\gamma-1}{c}\right]}{\Gamma[k(r+\gamma)+\gamma]}\right]\right\} .
\end{aligned}
$$

\section{Appendix C - Shannon entropy}

The Shannon entropy of a random variable $X$ with density function $f(x)$ is a measure of variation of the uncertainty. The Shannon entropy is given by

$$
\begin{aligned}
E\{-\log [f(X)]\} & =\log \left(1-\mathrm{e}^{-\lambda}\right)-\log (\alpha)-\log (\lambda)-(\alpha-1) \mathrm{E}\{\log [\mathrm{G}(\mathrm{X})]\} \\
& +\lambda E\left[G^{\alpha}(X)\right]-E\{\log [g(X)]\} \\
& =\log \left(1-\mathrm{e}^{-\lambda}\right)-\log (\alpha)-\log (\lambda)+(\alpha-1) \sum_{j, r=0}^{\infty} \frac{(r+1) v_{r}}{(j+1)(j+r+2)} \\
& +\lambda \sum_{j, r=0}^{\infty} \frac{(-1)^{j}(r+1) v_{r} \Gamma(\alpha+1)}{(j+r+1) j ! \Gamma(\alpha-j)}-E\{\log [g(X)]\} .
\end{aligned}
$$


We use the power series

$$
G^{\alpha}(X)=\left\{1-\left[1+\left(\frac{x}{s}\right)^{c}\right]^{-k}\right\}^{\alpha}=\sum_{j=0}^{\infty} \frac{(-1)^{j} \Gamma(\alpha+1)}{j ! \Gamma(\alpha-j)}\left[1+\left(\frac{x}{s}\right)^{c}\right]^{-j k}
$$

and

$$
\begin{aligned}
\log [G(X)] & =\sum_{j=0}^{\infty} \frac{(-1)^{j}}{j+1}[G(x)-1]^{j+1}=\sum_{j=0}^{\infty} \frac{(-1)^{j}}{j+1}\left\{-\left[1+\left(\frac{x}{s}\right)^{c}\right]^{-k}\right\}^{j+1} \\
& =\sum_{j=0}^{\infty} \frac{(-1)^{2 j+1}}{j+1}\left[1+\left(\frac{x}{s}\right)^{c}\right]^{-k(j+1)}=-\sum_{j=0}^{\infty} \frac{1}{j+1}\left[1+\left(\frac{x}{s}\right)^{c}\right]^{-k(j+1)}
\end{aligned}
$$

Next, we have

$$
\begin{aligned}
E\left[G^{\alpha}(X)\right] & =\sum_{r=0}^{\infty} v_{r} \int_{0}^{\infty} G^{\alpha}(x) g(x ; s, k(r+1), c) d x \\
& =c k s^{-c} \Gamma(\alpha+1) \sum_{j, r=0}^{\infty} \frac{(-1)^{j}(r+1) v_{r}}{j ! \Gamma(\alpha-j)} \int_{0}^{\infty} x^{c-1}\left[1+\left(\frac{x}{s}\right)^{c}\right]^{-k(j+r+1)-1} d x \\
& =c k s^{-c} \Gamma(\alpha+1) \sum_{j, r=0}^{\infty} \frac{(-1)^{j}(r+1) v_{r}}{j ! \Gamma(\alpha-j)} \frac{s^{c}}{c} \int_{0}^{\infty}(1+u)^{-k(j+r+1)-1} d x \\
& =\sum_{j, r=0}^{\infty} \frac{(-1)^{j} \Gamma(\alpha+1)(r+1) v_{r} \Gamma(\alpha+1)}{(j+r+1) j ! \Gamma(\alpha-j)}
\end{aligned}
$$

and

$$
\begin{aligned}
E\{\log [G(X)]\} & =\sum_{r=0}^{\infty} v_{r} \int_{0}^{\infty} \log G(x) g(x ; s, k(r+1), c) d x \\
& =-c k s^{-c} \sum_{j, r=0}^{\infty} \frac{(r+1) v_{r}}{j+1} \int_{0}^{\infty} x^{c-1}\left[1+\left(\frac{x}{s}\right)^{c}\right]^{-k(j+r+2)-1} d x \\
& =-c k s^{-c} \sum_{j, r=0}^{\infty} \frac{(r+1) v_{r}}{j+1} \frac{s^{c}}{c} \int_{0}^{\infty}(1+u)^{-k(j+r+2)-1} d x \\
& =-\sum_{j, r=0}^{\infty} \frac{(r+1) v_{r}}{(j+1)(j+r+2)} .
\end{aligned}
$$

Thus,

$$
\begin{aligned}
E\{-\log [f(X)]\} & =\log \left(1-\mathrm{e}^{-\lambda}\right)-\log (\alpha)-\log (\lambda)+(\alpha-1) \sum_{j, r=0}^{\infty} \frac{(r+1) v_{r}}{(j+1)(j+r+2)} \\
& +\lambda \sum_{j, r=0}^{\infty} \frac{(-1)^{j}(r+1) v_{r} \Gamma(\alpha+1)}{(j+r+1) j ! \Gamma(\alpha-j)}-E[\log g(X)] .
\end{aligned}
$$

\section{Appendix D - Information matrix}

The elements of the observed information matrix $J(\boldsymbol{\theta})$ for the parameters $(s, k, c, \alpha, \lambda)$ are 


$$
\begin{aligned}
& U_{s s}(\boldsymbol{\theta})=\frac{c}{s^{2}}+\frac{k c}{n s^{2}} \sum_{i=1}^{n} \frac{u_{i}^{2}\left(c+q_{i,-1}\right)}{q_{i,-2}} \\
& +\frac{k c(\alpha-1)}{n s^{2}} \sum_{i=1}^{n} \frac{u_{i}\left[c\left(1+k u_{i} q_{i,-k}-q_{i,-k}\right)-q_{i,-1}\left(q_{i,-k}-1\right)\right]}{q_{i,-2}\left(q_{i,-k}-1\right)^{2}} \\
& -\frac{k c \alpha \lambda}{n s^{2}} \sum_{i=1}^{n} \frac{u_{i} v_{i, \alpha}\left\{c\left[-k u_{i}\left(q_{i,-k}-\alpha\right)+\left(q_{i-k}-1\right)\right]+q_{i,-1}\left(q_{i,-k}-1\right)\right\}}{q_{i,-2}\left(q_{i,-k}-1\right)^{2}}, \\
& U_{s k}(\boldsymbol{\theta})=-\frac{c}{n s^{2}} \sum_{i=1}^{n} \frac{x_{i} u_{i}^{(c-1) / c}}{q_{i,-1}}+\frac{c(\alpha-1)}{n s} \sum_{i=1}^{n} \frac{u_{i}\left[1-q_{i,-k}+k q_{i,-k} \log \left(q_{i,-1}\right)\right]}{q_{i,-1}\left(q_{i,-k}-1\right)^{2}} \\
& -\frac{c \alpha \lambda}{n s} \sum_{i=1}^{n} \frac{u_{i} v_{i, \alpha}\left[-k \log \left(q_{i,-1}\right) u_{i}\left(q_{i,-k}-\alpha\right)+q_{i-k}-1\right]}{q_{i,-1}\left(q_{i,-k}-1\right)^{2}} \text {, } \\
& U_{s c}(\boldsymbol{\theta})=-\frac{1}{s}-\frac{k}{n s} \sum_{i=1}^{n} \frac{u_{i}\left[q_{i,-1}+c \log \left(u_{i}^{1 / c}\right)\right]}{q_{i,-2}} \\
& +\frac{k(\alpha-1)}{n s} \sum_{i=1}^{n} \frac{u_{i}\left\{c \log \left(u_{i}^{1 / c}\right)\left[1+k u_{i} q_{i,-k}-q_{i,-k}\right]-q_{i,-1}\left(q_{i,-k}-1\right)\right\}}{q_{i,-2}\left(q_{i,-k}-1\right)^{2}} \\
& -\frac{k c \alpha \lambda}{n s} \sum_{i=1}^{n} \frac{u_{i} v_{i, \alpha}\left\{\log \left(u_{i}^{1 / c}\right)\left[k u_{i}\left(q_{i,-k}-\alpha\right)-v_{i, 1}-q_{i,-1}\left(q_{i,-k}-1\right)\right]\right\}}{q_{i,-2}\left(q_{i,-k}-1\right)^{2}} \text {, } \\
& U_{s \alpha}(\boldsymbol{\theta})=-\frac{k c}{n s^{2}} \sum_{i=1}^{n} \frac{x_{i} u_{i}^{(c-1) / c} q_{i, k+1}}{v_{i, 1}}+\frac{k c \lambda}{n s} \sum_{i=1}^{n} \frac{u_{i} v_{i, \alpha}\left[1+\alpha \log \left(v_{i, 1}\right)\right]}{q_{i,-1}\left(q_{i,-k}-1\right)} \\
& U_{s \lambda}(\boldsymbol{\theta})=\frac{k c \alpha}{n s} \sum_{i=1}^{n} \frac{u_{i} q_{i, k+1}}{v_{i, \alpha-1}} \\
& U_{k k}(\boldsymbol{\theta})=-\frac{1}{k^{2}}-\frac{(\alpha-1)}{n} \sum_{i=1}^{n} \frac{q_{i,-k} \log ^{2}\left(q_{i,-1}\right)}{\left(q_{i,-k}-1\right)^{2}}+\frac{\alpha \lambda}{n} \sum_{i=1}^{n} \frac{v_{i, \alpha}\left(q_{i,-k}-\alpha\right) \log ^{2}\left(q_{i,-1}\right)}{\left(q_{i,-k}-1\right)^{2}} \\
& U_{k c}(\boldsymbol{\theta})=\frac{1}{n} \sum_{i=1}^{n} \frac{u_{i} \log \left(u_{i}^{1 / c}\right)}{q_{i,-1}}-\frac{(\alpha-1)}{n} \sum_{i=1}^{n} \frac{u_{i} \log \left(u_{i}^{1 / c}\right)\left[1-q_{i,-k}+k q_{i,-k} \log \left(q_{i,-1}\right)\right]}{q_{i,-1}\left(q_{i,-k}-1\right)^{2}} \\
& +\frac{k \alpha \lambda}{n} \sum_{i=1}^{n} \frac{u_{i} \log \left(u_{i}^{1 / c}\right) v_{i, \alpha} \log \left(q_{i,-1}\right)}{q_{i,-1}\left(q_{i,-k}-1\right)^{2}} \\
& U_{k \alpha}(\boldsymbol{\theta})=\frac{1}{n} \sum_{i=1}^{n} \frac{q_{i, k} \log \left(q_{i,-1}\right)}{v_{i, 1}}-\frac{\lambda}{n} \sum_{i=1}^{n} \frac{\log \left(q_{i,-1}\right) v_{i, \alpha}\left[1+\alpha \log \left(v_{i, 1}\right)\right]}{q_{i,-k}-1},
\end{aligned}
$$




$$
\begin{aligned}
& U_{k \lambda}(\boldsymbol{\theta})=-\frac{\alpha}{n} \sum_{i=1}^{n} q_{i, k} v_{i, \alpha} \log \left(q_{i,-1}\right), \\
& U_{c c}(\boldsymbol{\theta})=-\frac{1}{c^{2}}+\frac{k}{n} \sum_{i=1}^{n} \frac{u_{i} \log ^{2}\left(u_{i}^{1 / c}\right)}{q_{i,-2}} \\
& -\frac{k(\alpha-1)}{n} \sum_{i=1}^{n} \frac{u_{i} \log ^{2}\left(u_{i}^{1 / c}\right)\left[1+k u_{i} q_{i,-k}-q_{i,-k}\right]}{q_{i,-2}\left(q_{i,-k}-1\right)^{2}} \\
& -\frac{k \alpha \lambda}{n} \sum_{i=1}^{n} \frac{u_{i} \log ^{2}\left(u_{i}^{1 / c}\right) v_{i, \alpha}\left[-k u_{i}\left(q_{i,-k}-\alpha\right)+q_{i-k}-1\right]}{q_{i,-2}\left(q_{i,-k}-1\right)^{2}}, \\
& U_{c \alpha}(\boldsymbol{\theta})=\frac{k}{n} \sum_{i=1}^{n} \frac{u_{i} q_{i . k+1} \log \left(u_{i}^{1 / c}\right)}{v_{i, 1}}-\frac{k \lambda}{n} \sum_{i=1}^{n} \frac{u_{i} \log \left(u_{i}^{1 / c}\right) v_{i, \alpha}\left[1+\alpha \log \left(v_{i, 1}\right)\right]}{q_{i,-1}\left(q_{i,-k}-1\right)}, \\
& U_{c \lambda}(\boldsymbol{\theta})=-\frac{k \alpha}{n} \sum_{i=1}^{n} u_{i} q_{i, k+1} v_{i, \alpha-1} \log \left(u_{i}^{1 / c}\right), \\
& U_{\alpha \alpha}(\boldsymbol{\theta})=-\frac{1}{\alpha^{2}}-\frac{\lambda}{n} \sum_{i=1}^{n} v_{i, \alpha} \log ^{2}\left(v_{i, 1}\right), \\
& U_{\alpha \lambda}(\boldsymbol{\theta})=-\frac{1}{n} \sum_{i=1}^{n} v_{i, \alpha} \log \left(v_{i, 1}\right), \\
& U_{\lambda \lambda}(\boldsymbol{\theta})=-\frac{1}{\lambda^{2}}+\frac{\mathrm{e}^{\lambda}}{\left(\mathrm{e}^{\lambda}-1\right)^{2}},
\end{aligned}
$$

where $u_{i}, q_{i, k}, v_{i, \alpha}$ are given in Section 9.

\section{References}

Brito, E., Silva, G. O., Cordeiro, G. M., \& Demétrio, C. G. B. (2014). The gamma Burr XII distribution: Theory and Practice. Journal of Data Science, In Press.

Chen, G., \& Balakrishnan, N. (1995). A general purpose approximate goodness-of-fit test. Journal of Quality Technology, 27, 154-161.

Cordeiro, G. M., Ortega, E. M. M., Hamedani, G. G., \& Garcia, D. A. (2012). The McBurr XII Model. Accepted.

El-Bassiouny, A. H., \& Abdo, N. F. (2010). Reliability properties of seven parameters Burr XII distribution. Comput Meth Sci Tech, 16, 127-133. http://dx.doi.org/10.12921/cmst.2010.16.02.127-133

Gradshteyn, I. S., \& Ryzhik, I. M. (2000). Table of Integrals, Series and Products. Academic Press, San Diego.

Hosking, J. R. M. (1990). L-moments: analysis and estimation of distributions using linear combinations of order statistics. Journal of the Royal Statistical Society B, 52, 105-124.

Jayakumar, K., \& Mathew, T. (2008). On a generalization to Marshall-Olkin scheme and its application to Burr type XII distribution. Stat Pap, 49, 421-439. http://dx.doi.org/10.1007/s00362-006-0024-5 
Kenney, J. F., \& Keeping, E. S. (1962). Mathematics of Statistics, 3rd ed., Part 1. New Jersey.

Murthy, D. N. P., Xie. M., \& Jiang, R. (2004). Weibull Models, John Wiley and Sons, New Jersey.

Parnaiba, P. F. P., Ortega, E. M. M., Cordeiro, G. M., \& Pescim, R. R. (2011). The beta Burr XII distribution with application to lifetime data. Computational Statistics and Data Analysis, 55, 1118-1136. http://dx.doi.org/10.1016/j.csda.2010.09.009

Parnaiba, P. F. P., Ortega, E. M. M., Cordeiro, G. M., \& Pascoa, M. A. R. (2012). The Kumaraswamy Burr XII distribution: Theory and Practice. Journal of Statistical Computation and Simulation, 82, 1-27.

Prudnikov, A. P., Brychkov, Y. A., \& Marichev, O.I. (1986). Integrals and Series, volume 1. Gordon and Breach Science Publishers, Amsterdam.

Prudnikov, A. P., Brychkov, Y. A., \& Marichev, O. I. (1992). Integrals and Series, volume 4. Gordon and Breach Science Publishers, Amsterdam.

Ramos, M. W. A., Percontini, A., Cordeiro, G. M., \& Silva, R. V. (2015). The Burr XII Negative Binomial Distribution with Applications to Lifetime Data. International Journal of Statistics and Probability, 4, 109125. http://dx.doi.org/10.5539/ijsp.v4n1p109

R Development Core Team. (2012). R: A Language and Environment for Statistical Computing. R Foundation for Statistical Computing: Vienna.

Rényi, A. (1961). On measures of entropy and information. In: Proceedings of the 4th Berkeley Symposium on Mathematical Statistics and Probability, Volume I, pp. 547-561. University of California Press: Berkeley.

Ristic, M. M., \& Nadarajah, S. (2012). A new lifetime distribution. Journal of Statistical Computation and Simulation, 81, 1-16.

Shao, Q. (2004a). Notes on maximum likelihood estimation for the three-parameter Burr XII distribution . Computational Statistics and Data Analysis, 45, 675-687. http://dx.doi.org/10.1016/S0167-9473(02)00367-5

Shao, Q., Wong, H., \& Xia, J. (2004b). Models for extremes using the extended three parameter Burr XII system with application to flood frequency analysis. Hydrological Sciences Journal des Sciences Hydrologiques, 49, 685-702. http://dx.doi.org/10.1623/hysj.49.4.685.54425

Soliman, A. A. (2005). Estimation of Parameters of Life From Progressively Censored Data Using Burr-XII Model. IEEE Transactions on Reliability, 54, 34-42. http://dx.doi.org/10.1109/TR.2004.842528

Zimmer, W. J., Keats, J. B., \& Wang, F. K. (1998). The Burr XII distribution in reliability analysis. Journal of Quality Technology, 30, 386-94.

\section{Copyrights}

Copyright for this article is retained by the author(s), with first publication rights granted to the journal.

This is an open-access article distributed under the terms and conditions of the Creative Commons Attribution license (http://creativecommons.org/licenses/by/3.0/). 